\title{
Confronting SUSY SO(10) with updated Lattice and Neutrino data
}

\author{
Thomas Deppisch, ${ }^{a}$ Stefan Schacht ${ }^{b, c}$ and Martin Spinrath ${ }^{d, e}$ \\ ${ }^{a}$ Institut für Theoretische Teilchenphysik, Karlsruhe Institute of Technology, \\ Engesserstraße 7, D-76131 Karlsruhe, Germany \\ ${ }^{b}$ Department of Physics, LEPP, Cornell University, \\ Ithaca, NY 14853, U.S.A. \\ ${ }^{c}$ Dipartimento di Fisica, Università di Torino 8 INFN, \\ Sezione di Torino, I-10125 Torino, Italy \\ ${ }^{d}$ Department of Physics, National Tsing Hua University, \\ Hsinchu 30013, Taiwan \\ e Physics Division, National Center for Theoretical Sciences, \\ Hsinchu 30013, Taiwan \\ E-mail: thomas.deppisch@kit.edu, ss3843@cornell.edu, \\ spinrath@phys.nthu .edu.tw
}

ABSTRACT: We present an updated fit of supersymmetric $\mathrm{SO}(10)$ models to quark and lepton masses and mixing parameters. Including latest results from lattice QCD determinations of quark masses and neutrino oscillation data, we show that fits neglecting supersymmetric threshold corrections are strongly disfavoured in our setup. Only when we include these corrections we find good fit points. We present $\chi^{2}$-profiles for the threshold parameters, which show that in our setup the thresholds related to the third generation of fermions exhibit two rather narrow minima.

Keywords: GUT, Neutrino Physics, Quark Masses and SM Parameters

ArXiv EPrint: 1811.02895 


\section{Contents}

1 Introduction 1

2 Fitting GUT scale Yukawa couplings to fermion masses 2

2.1 Yukawa couplings in SO(10) GUTs 3

2.2 RGE running from the GUT scale to the SUSY scale 4

2.3 Fermion masses at the SUSY scale 4

$\begin{array}{lll}2.4 & \text { SM vs. MSSM Yukawa couplings } & 6\end{array}$

2.5 Fitting procedure 8

3 Results of the SUSY SO(10) fit to flavour data 9

3.1 Comparison of the global minima 9

$\begin{array}{ll}3.2 & \text { Likelihood profiles for threshold corrections } \\ \end{array}$

4 Summary and conclusions $\quad 14$

$\begin{array}{ll}\text { A SM observables at various scales } & 16\end{array}$

$\begin{array}{ll}\text { B GUT scale parameters for the global minima } & 17\end{array}$

$\begin{array}{ll}\text { B.1 Global minima without SUSY threshold corrections } & 17\end{array}$

$\begin{array}{lll}\text { B.2 Global minima with SUSY threshold corrections } & 18\end{array}$

$\begin{array}{lll}\text { B.3 Local minima with SUSY threshold corrections } & 19\end{array}$

\section{Introduction}

Since the advent of Maxwell's theory of electromagnetism it is a common dream in physics to unify all interactions into one single theory. This dream is particularly persistent in particle physics where Grand Unified Theories (GUTs) - which unify three of the fundamental forces of nature - have been a major guiding principle in the last couple of decades. To be precise in this paper we will focus on GUTs based on the $\mathrm{SO}(10)$ gauge group first proposed in the 1970s [1, 2]. This choice contains all the essential features interpreted as hints towards unification, like charge quantization, anomaly cancellation or smallness of neutrino masses.

$\mathrm{SO}(10)$ GUTs work especially well in the context of low energy supersymmetry (SUSY) which helps significantly to unify the gauge couplings, see, e.g., [3]. In fact, supersymmetry is part of the dream of unification as it unifies the concepts of bosons and fermions or the concepts of matter and forces. It also provides an elegant mechanism to stabilize mass hierarchies and provides a dark matter candidate on top.

In this paper though we will not discuss all features and aspects of SUSY SO(10) models in general. We focus on the Yukawa sector, which is interesting because this 
sector is constrained by low energy observables, namely the observed fermion masses and mixing parameters. There has been some tremendous progress in the last couple of years in particular for light quark masses from lattice computations and neutrino masses and mixing from oscillation experiments. We will use this updated information to provide an updated fit to a minimal SUSY SO(10) Yukawa sector.

There is a long history of fitting $\mathrm{SO}(10)$ to fermion masses, see, e.g., [8-52]. Some works focussed on particular $\mathrm{SO}(10)$ models, while others focussed on a general $\mathrm{SO}(10)$ Yukawa sector only. These efforts have always been a difficult task due to the large number of parameters and observables. For that reason many groups had to resort to some simplifications or estimates. For instance, the recent fits by Dueck and Rodejohann [50] neglect in the SUSY case threshold corrections which are known to give sizeable, important corrections, e.g., refs. [4-7, 38, 44, 53, 54]. One of the main improvements of our work compared to previous studies is to include these threshold corrections in terms of three parameters and to provide $\chi^{2}$-profiles for them for the first time, to our knowledge.

Our paper is organised as follows: in section 2 we describe in detail how we match the $\mathrm{SO}(10)$ Yukawa couplings at the high scale to the fermion masses and mixing parameters at low energies including the fit procedure. In section 3 we discuss our results. First we present the global minima of our fits and the pulls to identify which observables are potentially driving some tensions of the data with our setup. Then we present the $\chi^{2}$-profiles for the SUSY threshold corrections before we summarise and conclude in section 4 . We provide additional information in two appendices. Appendix A gives additional numerical values for Standard Model (SM) observables, and appendix B the values of the GUT scale fit parameters at the global and local minima.

\section{Fitting GUT scale Yukawa couplings to fermion masses}

Besides the unification of the gauge sector, SO(10) GUTs also predict the unification of the Yukawa sector by arranging all matter fields in the spinorial $\mathbf{1 6}$ representation. Of course, these constraints hold only at the GUT scale and effects of GUT breaking and of the renormalisation group equations (RGEs) will alter the relations among the Yukawa couplings.

Below the GUT scale, important physics happens around the seesaw scale where the heavy right-handed neutrinos are integrated out one after another. Moreover, SUSY breaking effects have to be taken into account in supersymmetric GUTs. Especially for large values of $\tan \beta$, threshold corrections from SUSY particles have sizeable effects [4-7]. In our analysis, we choose the matching scale between the SM and its minimal supersymmetric extension $(\mathrm{MSSM})$ to be $M_{\mathrm{SUSY}}=1 \mathrm{TeV}$. At this scale we define as well the $\chi^{2}$-function to fit the GUT parameters to the SM Yukawa couplings. Therefore we first need to evolve the fermion masses and mixing parameters to $M_{\mathrm{SUSY}}$ using the SM $\beta$-functions. We describe the procedure in greater detail in the following. 


\subsection{Yukawa couplings in SO(10) GUTs}

In this work we restrict ourselves to renormalisable $\mathrm{SO}(10)$ GUTs. This has a stronger predictive power than SU(5) or non-renormalisable GUTs. Furthermore we assume the standard embedding of the matter fields into the spinorial 16 of $\mathrm{SO}(10)$. This restricts the Higgs representations relevant for fermion masses to a 10, 120 and/or $\overline{\mathbf{1 2 6}}$ of $\mathrm{SO}(10)$.

In SUSY SO(10), the most general renormalisable superpotential describing Yukawa interactions is then given by

$$
\mathcal{W}=Y_{10}^{i j} \mathbf{1 6}_{i} \cdot \mathbf{1 6}_{j} \cdot \mathbf{1 0}_{H}+Y_{120}^{i j} \mathbf{1 6}_{i} \cdot \mathbf{1 6}_{j} \cdot \mathbf{1 2 0}_{H}+Y_{126}^{i j} \mathbf{1 6}_{i} \cdot \mathbf{1 6}_{j} \cdot \overline{\mathbf{1 2 6}}_{H},
$$

where the $\mathbf{1 6}_{i}$ are the three generations of matter fields with flavour indices $i, j=1,2,3$. The fields $\mathbf{1 0}_{H}, \mathbf{1 2 0}_{H}$ and $\overline{\mathbf{1 2 6}}_{H}$ are GUT representations containing Higgs fields and we assume maximum one of each. Due to the $\mathrm{SO}(10)$ gauge structure the Yukawa matrices $Y_{10}^{i j}$ and $Y_{126}^{i j}$ are symmetric while $Y_{120}^{i j}$ is antisymmetric in the flavour indices $i$ and $j$. Without loss of generality we apply an unphysical flavour rotation to choose $Y_{10}$ real and diagonal.

After breaking $\mathrm{SO}(10)$ at the high scale $M_{\mathrm{GUT}}=1.353 \times 10^{16} \mathrm{GeV}$, given by gauge coupling unification, ${ }^{1}$ the Yukawa matrices are matched to the MSSM Yukawa matrices $Y_{x}, x=u, d, e, \nu$, the Majorana mass matrix for the right handed neutrinos $M$ and the Wilson coefficient of the Weinberg operator $\kappa$, see for example ref. [50]

$$
\begin{aligned}
Y_{u} & =r\left(Y_{10}+s Y_{126}+\mathrm{i} t_{u} Y_{120}\right), \\
Y_{d} & =Y_{10}+Y_{126}+\mathrm{i} t_{d} Y_{120}, \\
Y_{\nu} & =r\left(Y_{10}-3 s Y_{126}+\mathrm{i} t_{\nu} Y_{120}\right), \\
Y_{e} & =Y_{10}-3 Y_{126}+\mathrm{i} t_{e} Y_{120}, \\
M & =r_{R} Y_{126}, \\
\kappa & =r_{L} Y_{126} .
\end{aligned}
$$

The parameters $s, t_{u}, t_{d}, t_{\nu}, t_{e}$ correspond to the mixing of GUT scale Higgs doublets into the MSSM Higgs doublets $H_{u}, H_{d}$ whereas $r, r_{R}, r_{L}$ correspond to the vacuum expectation values (vevs) of the GUT scale Higgs fields and can be chosen real without loss of generality.

A minimal realistic choice omits the $\mathbf{1 2 0}$ representation (i.e., the parameters $t_{u}, t_{d}$, $\left.t_{\nu}, t_{e}\right)$ and $r_{L}$. Previous fits to fermion observables have shown that the $\mathbf{1 2 0}$ does not significantly improve the fit to fermion masses in the SM, e.g., ref. [50], so we will not include it here $\left(Y_{120}=0\right)$. Furthermore, in ref. [34] it was argued that while a generic fit to fermion masses prefers a mix of type-I seesaw (parametrised by $r_{R}$ ) and type-II seesaw (parametrised by $r_{L}$ ), a fit which includes the GUT potential favours scenarios where typeI is dominant over type-II. Hence, we will assume $r_{L}=0$. Thus we end up with a set of 19 GUT scale parameters: $Y_{10}$ (three parameters), $Y_{126}$ (twelve parameters), $r_{R}$ (one parameter), $r$ (one parameter) and $s$ (two parameters).

Before we discuss our numerical implementation and the results we want to give a few comments on Yukawa couplings from higher-dimensional operators. In this study we explicitly assume that no higher-dimensional operators correct the Yukawa coupling relations

\footnotetext{
${ }^{1}$ For details see appendix B.
} 
beyond the ones present at renormalisable level. Especially, in the context of supergravity one might expect higher-dimensional operators to be present in the superpotential suppressed by powers of $M_{\mathrm{GUT}} / M_{\text {Planck }}=\mathcal{O}(0.01)$. Such higher-dimensional operators could alter the Yukawa relations if they have a non-trivial gauge structure.

It is an open question if quantum gravity introduces GUT non-singlets at the Planck scale which couple to ordinary matter. The presence of non-singlets would imply that quantum gravity knows something about flavour. If it would be flavour-blind all Yukawa couplings should be at least of order $M_{\mathrm{GUT}} / M_{\text {Planck }}=\mathcal{O}(0.01)$. However, this is not the case unless there are some large cancellations at work. Hence, we interpret the smallness of the electron mass as an indication that these higher-dimensional operators are negligibly small and we do not consider them here. Nevertheless, in a supersymmetric GUT theory of flavour they can be the dominant operators and very sensible, for a recent review, see, e.g., ref. [55].

\subsection{RGE running from the GUT scale to the SUSY scale}

The values of the Yukawa couplings at different energy scales are related by the renormalisation group equations via

$$
Y\left(\mu_{2}\right)=Y\left(\mu_{1}\right)+\int_{\log \mu_{1}}^{\log \mu_{2}} \mathrm{~d} \log \mu \beta_{Y}(\mu),
$$

with the beta function being defined as $\beta_{Y}(\mu)=\mathrm{d} Y / \mathrm{d} \log \mu$. At one- and two-loop level they are well-known for both the SM and the MSSM with additional right-handed neutrinos, see, e.g., ref. [56]. We also include the running of the Weinberg operator at the one-loop level.

For every point in the GUT parameter space the RGEs have to be solved, i.e. numerically integrated. For a correct treatment of the seesaw mechanism, we consecutively integrate out the right-handed neutrinos as described in ref. [57] and implemented in the Mathematica package REAP presented therein. REAP provides a useful tool for cross checking our calculations. We implement the numerical procedure in $\mathrm{C}++$ using the template libraries odeint [58] and eigen3 [59]. This speeds up the calculation of the RGE running by up to a factor of 100 compared to the Mathematica version of REAP on our test desktop machine (Intel i5-4590 CPU, 3.30 GHz).

\subsection{Fermion masses at the SUSY scale}

Our low scale input for the quark masses and $\alpha_{s}$ is given in table 1. We perform the QCD RGE evolution to $M_{Z}=91.1876 \mathrm{GeV}$ [61] and the matching from 4 to 5 as well as from 5 to 6 active flavours with the Mathematica package RunDec [62] to four-loop accuracy. Our results are shown in table 2. Errors from variation of the bottom and top quark scales are negligible compared to the experimental uncertainties. The Yukawa couplings at $M_{Z}$ can be obtained from $y=m / v$, with $v=174.104 \mathrm{GeV}$. As additional input at $M_{Z}$, we use the values for the gauge couplings $g_{1}$ and $g_{2}$ as well as the values for the lepton Yukawa couplings from ref. [63]. Regarding the input for the CKM matrix, we use the ICHEP2016 update from CKMfitter [64]. Corresponding results from UTfit are in good agreement [65]. 


\begin{tabular}{|ccc|}
\hline Input & \multicolumn{2}{c|}{ Value } \\
\hline$m_{u}(2 \mathrm{GeV})$ & $2.36(24)$ & $\mathrm{MeV}$ \\
$m_{d}(2 \mathrm{GeV})$ & $5.03(26)$ & $\mathrm{MeV}$ \\
$m_{s}(2 \mathrm{GeV})$ & $93.9(1.1)$ & $\mathrm{MeV}$ \\
$m_{c}(3 \mathrm{GeV})$ & $996(25)$ & $\mathrm{MeV}$ \\
$\bar{m}_{b}\left(\bar{m}_{b}\right)$ & $4.190(21)$ & $\mathrm{GeV}$ \\
$\alpha_{s}^{(5)}\left(M_{Z}\right)$ & \multicolumn{2}{c}{$0.1182(12)$} \\
\hline
\end{tabular}

Table 1. Input data for SM parameters in the $\overline{\mathrm{MS}}$ scheme from lattice QCD determinations with $N_{f}=2+1+1$ taken from ref. [60].

\begin{tabular}{|cc|}
\hline Result at $M_{Z}$ & Value \\
\hline$m_{u}^{(6)}\left(M_{Z}\right)$ & $1.36(15) \mathrm{MeV}$ \\
$m_{d}^{(6)}\left(M_{Z}\right)$ & $2.90(11) \mathrm{MeV}$ \\
$m_{s}^{(6)}\left(M_{Z}\right)$ & $54.05(63) \mathrm{MeV}$ \\
$m_{c}^{(6)}\left(M_{Z}\right)$ & $635(16) \mathrm{MeV}$ \\
$m_{b}^{(6)}\left(M_{Z}\right)$ & $2.866(14) \mathrm{GeV}$ \\
$m_{t}^{(6)}\left(M_{Z}\right)$ & $172.3(1.5) \mathrm{GeV}$ \\
$\alpha_{s}^{(6)}\left(M_{Z}\right)$ & $0.1170(12)$ \\
\hline
\end{tabular}

Table 2. Results of the QCD RG evolution for six active flavours and a top-quark pole mass $m_{t, \text { pole }}=174.2 \pm 1.4 \mathrm{GeV}[61]$.

In order to perform the RGE evolution from $M_{Z}$ to $M_{\mathrm{SUSY}}$, we use the two-loop RGEs for the SM Yukawas and gauge couplings [66]. For solving the RGEs at two-loop also the Higgs quartic coupling is needed which we get from the PDG average of the Higgs mass measurement $m_{\text {Higgs }}=125.09 \pm 0.24 \mathrm{GeV}$ [61]. For the off-diagonal elements of the Yukawa matrices we use the standard parametrisation of the PDG. Further details can be found in appendix A.

We treat the uncertainties of the experimental data as Gaussian and symmetrise them arithmetically where necessary. For the propagation of uncertainties we sample normally distributed random numbers at $M_{Z}$. Each sample point is then evolved to the respective energy scale. There, we fit a normal distribution to the set of sample points. Thus, also non-linear effects, especially the influence of the top quark Yukawa coupling, and interdependencies in the RGE running are taken into account. This has the effect that the relative errors are larger at higher energy scales due to the error of the top quark mass measurement. 


\begin{tabular}{|c|c|c|c|c|}
\hline & $M_{Z}$ & $1 \mathrm{TeV}$ & $3 \mathrm{TeV}$ & $10 \mathrm{TeV}$ \\
\hline$g_{1}$ & $0.461425_{-0000043}^{+0.000044}$ & $0.467773 \pm 0.000045$ & $0.470766 \pm 0.000046$ & $0.474109 \pm 0.000047$ \\
\hline$g_{2}$ & $0.65184_{-0.00017}^{+0.00018}$ & $0.63935 \pm 0.00016$ & $0.63383 \pm 0.00016$ & $0.62792 \pm 0.00016$ \\
\hline$g_{3}$ & $1.2127 \pm 0.0061$ & $1.0549 \pm 0.0040$ & $1.0009 \pm 0.0034$ & $0.9503 \pm 0.0029$ \\
\hline$y_{u} / 10^{-6}$ & $7.80 \pm 0.86$ & $6.73 \pm 0.74$ & $6.37 \pm 0.70$ & $6.03 \pm 0.66$ \\
\hline$y_{c} / 10^{-3}$ & $3.646 \pm 0.091$ & $3.147 \pm 0.79$ & $2.976 \pm 0.074$ & $2.816 \pm 0.071$ \\
\hline$y_{t}$ & $0.9897 \pm 0.0086$ & $0.8723 \pm 0.0088$ & $0.8317 \pm 0.0088$ & $0.7934 \pm 0.0089$ \\
\hline$y_{d} / 10^{-5}$ & $1.663 \pm 0.064$ & $1.438 \pm 0.056$ & $1.361 \pm 0.053$ & $1.289 \pm 0.050$ \\
\hline$y_{s} / 10^{-4}$ & $3.104 \pm 0.036$ & $2.685 \pm 0.032$ & $2.541 \pm 0.030$ & $2.407 \pm 0.029$ \\
\hline$y_{b} / 10^{-2}$ & $1.646 \pm 0.0082$ & $1.3940 \pm 0.0079$ & $1.3091 \pm 0.0071$ & $1.2303 \pm 0.0070$ \\
\hline$y_{e} / 10^{-6}$ & $2.794745_{-0.000016}^{+0.000015}$ & $2.8491 \pm 0.0022$ & $2.8659 \pm 0.0031$ & $2.8800 \pm 0.0040$ \\
\hline$y_{\mu} / 10^{-4}$ & $5.899863_{-0.000018}^{+0.000019}$ & $6.0146 \pm 0.0046$ & $6.0501 \pm 0.0065$ & $6.080 \pm 0.0086$ \\
\hline$y_{\tau} / 10^{-2}$ & $1.002950_{-0.000091}^{+0.000090}$ & $1.02246 \pm 0.00078$ & $1.0285 \pm 0.0011$ & $1.0336 \pm 0.0014$ \\
\hline$\theta_{12}^{q}$ & $0.22704_{-0.00029}^{+0.00030}$ & $0.22704 \pm 0.00029$ & $0.22704 \pm 0.00029$ & $0.22705 \pm 0.00029$ \\
\hline$\theta_{13}^{q} / 10^{-3}$ & $3.71_{-0.14}^{+0.13}$ & $3.79 \pm 0.14$ & $3.82 \pm 0.14$ & $3.85 \pm 0.14$ \\
\hline$\theta_{23}^{q} / 10^{-2}$ & $4.181_{-0.067}^{+0.047}$ & $4.270 \pm 0.058$ & $4.303 \pm 0.058$ & $4.337 \pm 0.059$ \\
\hline$\delta_{\mathrm{CP}}^{q}$ & $1.143_{-0.011}^{+0.011}$ & $1.143 \pm 0.011$ & $1.143 \pm 0.011$ & $1.143 \pm 0.011$ \\
\hline
\end{tabular}

Table 3. SM observables in the $\overline{\mathrm{MS}}$ scheme at $M_{Z}, 1 \mathrm{TeV}, 3 \mathrm{TeV}$ and $10 \mathrm{TeV}$. Quark masses and $\alpha_{s}$ are taken from lattice determinations [60] and evolved to $M_{Z}$ with RunDec [62]. All quark Yukawa couplings and $\alpha_{s}$ correspond to six active flavours. Lepton Yukawas and electroweak gauge couplings are taken from ref. [63], CKM parameters from the ICHEP 2016 update of ref. [64]. Note that we use the GUT normalisation for $g_{1}$. The running between $M_{Z}$ and 1,3 and $10 \mathrm{TeV}$ is performed using the SM RGEs at two-loop. Gaussian errors are propagated applying Bayes' theorem.

For our global fit we set $M_{\mathrm{SUSY}}=1 \mathrm{TeV}$. However, for reference, in table 3 we additionally list also the SM Yukawa and gauge couplings in the $\overline{\mathrm{MS}}$ scheme at $M_{Z}, 3 \mathrm{TeV}$ and $10 \mathrm{TeV}$ for reference. The same results converted to the $\overline{\mathrm{DR}}$ scheme can be found in table 4 .

\subsection{SM vs. MSSM Yukawa couplings}

At the SUSY scale (which we set to $1 \mathrm{TeV}$ ) two important things happen at the same time. First of all, loop calculations in the SM are usually done in the $\overline{\mathrm{MS}}$ scheme which is unsuitable for supersymmetry. For supersymmetry the $\overline{\mathrm{DR}}$ scheme is preferred. We will use two-loop RGEs so we have to perform the matching at the one-loop level. The relevant formulae for gauge couplings and Yukawa couplings are [67]

$$
\begin{aligned}
g_{\overline{\mathrm{MS}}} & =g_{\overline{\mathrm{DR}}}\left(1-\frac{g^{2}}{96 \pi^{2}} C(G)\right), \\
{\left[Y_{\overline{\mathrm{MS}}}^{i}\right]^{j k} } & =\left[Y_{\overline{\mathrm{DR}}}^{i}\right]^{j k}\left(1+\frac{g_{a}^{2}}{32 \pi^{2}}\left(C_{a}\left(r_{i}\right)+C_{a}\left(r_{j}\right)-2 C_{a}\left(r_{k}\right)\right)\right),
\end{aligned}
$$




\begin{tabular}{|ccccc|}
\hline & $M_{Z}$ & $1 \mathrm{TeV}$ & $3 \mathrm{TeV}$ & $10 \mathrm{TeV}$ \\
\hline$g_{1}$ & $0.461425_{-0000043}^{+0.000044}$ & $0.467773 \pm 0.000045$ & $0.470766 \pm 0.000046$ & $0.474109 \pm 0.000047$ \\
$g_{2}$ & $0.65243 \pm 0.00018$ & $0.63990 \pm 0.00017$ & $0.63437 \pm 0.00016$ & $0.62844 \pm 0.00016$ \\
$g_{3}$ & $1.2185 \pm 0.0062$ & $1.0587 \pm 0.0040$ & $1.00415 \pm 0.0034$ & $0.9530 \pm 0.0029$ \\
\hline$y_{u} / 10^{-6}$ & $7.71 \pm 0.85$ & $6.68 \pm 0.74$ & $6.32 \pm 0.70$ & $5.99 \pm 0.66$ \\
$y_{c} / 10^{-3}$ & $3.60 \pm 0.090$ & $3.120 \pm 0.078$ & $2.954 \pm 0.074$ & $2.797 \pm 0.071$ \\
$y_{t}$ & $0.9785 \pm 0.0086$ & $0.8651 \pm 0.0087$ & $0.8255 \pm 0.0087$ & $0.7881 \pm 0.0088$ \\
\hline$y_{d} / 10^{-5}$ & $1.64441 \pm 0.064$ & $1.426 \pm 0.056$ & $1.351 \pm 0.053$ & $1.281 \pm 0.050$ \\
$y_{s} / 10^{-4}$ & $3.06979 \pm 0.036$ & $2.663 \pm 0.032$ & $2.523 \pm 0.030$ & $2.392 \pm 0.029$ \\
$y_{b} / 10^{-2}$ & $1.6274 \pm 0.0082$ & $1.3825 \pm 0.0073$ & $1.2995 \pm 0.0071$ & $1.2224 \pm 0.0070$ \\
\hline$y_{e} / 10^{-6}$ & $2.796719 \pm 0.000016$ & $2.8510 \pm 0.0022$ & $2.8677 \pm 0.0031$ & $2.8818 \pm 0.0041$ \\
$y_{\mu} / 10^{-4}$ & $5.904029 \pm 0.000019$ & $6.0186 \pm 0.0046$ & $6.0540 \pm 0.0065$ & $6.0836 \pm 0.0086$ \\
$y_{\tau} / 10^{-2}$ & $1.003658 \pm 0.000091$ & $1.0231 \pm 0.0078$ & $1.0292 \pm 0.0011$ & $1.0342 \pm 0.0015$ \\
\hline$\theta_{12}^{q}$ & $0.22704_{-0.00029}^{+0.00030}$ & $0.22704 \pm 0.00029$ & $0.22704 \pm 0.00029$ & $0.22705 \pm 0.00029$ \\
$\theta_{13}^{q} / 10^{-3}$ & $3.71_{-0.14}^{+0.13}$ & $3.79 \pm 0.14$ & $3.82 \pm 0.14$ & $3.85 \pm 0.14$ \\
$\theta_{23}^{q} / 10^{-2}$ & $4.181_{-0.067}^{+0.047}$ & $4.270 \pm 0.058$ & $4.303 \pm 0.058$ & $4.337 \pm 0.059$ \\
$\delta_{\mathrm{CP}}^{q}$ & $1.143_{-0.011}^{+0.011}$ & $1.143 \pm 0.011$ & $1.143 \pm 0.011$ & $1.143 \pm 0.011$ \\
\hline
\end{tabular}

Table 4. SM observables in the $\overline{\mathrm{DR}}$ scheme at $M_{Z}, 1 \mathrm{TeV}, 3 \mathrm{TeV}$ and $10 \mathrm{TeV}$. The results at $1 \mathrm{TeV}$ are included as input in our $\mathrm{SO}(10)$ fit. Quark masses and $\alpha_{s}$ are taken from lattice determinations [60] and evolved to $M_{Z}$ with RunDec [62]. All quark Yukawa couplings and $\alpha_{s}$ correspond to six active flavours. Lepton Yukawa couplings and electroweak gauge couplings are taken from ref. [63], CKM parameters from the ICHEP 2016 update of ref. [64]. Note that we use the GUT normalisation for $g_{1}$. The running between $M_{Z}$ and 1,3 and $10 \mathrm{TeV}$ is performed using the SM RGEs at two-loop. Gaussian errors are propagated applying Bayes' theorem. All gauge couplings and Yukawa couplings have been converted to $\overline{\mathrm{DR}}$ at the given scale according to ref. [67].

with $C(G)=N$ and $C(r)=\left(N^{2}-1\right) / 2 N$ for $\mathrm{SU}(N)$. In the MSSM the matching conditions for the Yukawa matrices then read

$$
\begin{aligned}
& Y_{\overline{\mathrm{MS}}}^{u}=Y_{\overline{\mathrm{DR}}}^{u}\left(1+\frac{1}{32 \pi^{2}}\left(-\frac{g_{1}^{2}}{60}-\frac{3 g_{2}^{2}}{4}+\frac{8 g_{3}^{2}}{3}\right)\right), \\
& Y_{\overline{\mathrm{MS}}}^{d}=Y_{\overline{\mathrm{DR}}}^{d}\left(1+\frac{1}{32 \pi^{2}}\left(-\frac{13 g_{1}^{2}}{60}-\frac{3 g_{2}^{2}}{4}+\frac{8 g_{3}^{2}}{3}\right)\right), \\
& Y_{\overline{\mathrm{MS}}}^{e}=Y_{\overline{\mathrm{DR}}}^{e}\left(1+\frac{1}{32 \pi^{2}}\left(\frac{9 g_{1}^{2}}{20}-\frac{3 g_{2}^{2}}{4}\right)\right) .
\end{aligned}
$$

The second thing is that the Yukawa couplings in the SM and the MSSM are not the same, not even on tree-level since the MSSM is a two Higgs doublet model. This introduces a dependence on $\tan \beta$, the ratio of the two Higgs vevs. Beyond that also finite one-loop corrections have to be taken into account in the matching. In particular there are certain pieces which are enhanced by $\tan \beta$ [4-7] and thus can easily make changes of $\mathcal{O}(10 \%)$ or even larger on the Yukawa couplings. 
The approach we will take here is well documented, for instance, in refs. [54, 68, 69], see also ref. [63], so that we will not go into much detail here. Note that we include the $\tan \beta$ enhanced parts only. This allows a simple parametrisation of the SUSY threshold corrections in terms of three parameters, $\epsilon_{b}, \epsilon_{q}$ and $\epsilon_{l}$, only using some rather mild, plausible assumptions on the SUSY breaking parameters. To be more precise we assume that the squark and slepton mass matrices are very close to being proportional to the unit matrix and that the trilinear couplings are hierarchical and dominated by the 3-3 element.

For the up-type quarks we can use the tree-level matching relation for SM and MSSM Yukawa couplings in the $\overline{\mathrm{DR}}$ scheme,

$$
\sin \beta Y_{\mathrm{MSSM}}^{u}=Y_{\mathrm{SM}}^{u},
$$

since their threshold corrections are proportional to $\cot \beta \ll 1$.

For the Yukawa couplings of the charged leptons and down-type quarks there are $\tan \beta$ enhanced threshold corrections in the matching formulas

$$
\begin{aligned}
\cos \beta\left(1+\epsilon_{l} \tan \beta\right) Y_{\mathrm{MSSM}}^{e} & =Y_{\mathrm{SM}}^{e}, \\
\cos \beta \operatorname{diag}\left(1+\epsilon_{q} \tan \beta, 1+\epsilon_{q} \tan \beta, 1+\epsilon_{b} \tan \beta\right) Y_{\mathrm{MSSM}}^{d} & =Y_{\mathrm{SM}}^{d},
\end{aligned}
$$

where we have used the conventions as in ref. [69] but we defined here $\epsilon_{b} \equiv \epsilon_{A}+\epsilon_{q}$.

Note that $Y_{\text {MSSM }}^{e}$ and $Y_{\text {MSSM }}^{d}$ are not diagonal in our approach and we determine the corresponding low energy mixing parameters from these matrices after the matching.

In the following, if we do not specify SM or MSSM we refer to MSSM $\overline{\mathrm{DR}}$ quantities.

\subsection{Fitting procedure}

For every point in the GUT parameter space that is scanned over we extract the fermion observables $\mathcal{O}_{i}^{\text {theo }}$ at $1 \mathrm{TeV}$ and compare it to the experimental data $\mathcal{O}_{i}^{\exp }$ with the $\chi^{2}$-function

$$
\chi^{2}=\sum_{i=1}^{15}\left(\frac{\mathcal{O}_{i}^{\text {theo }}-\mathcal{O}_{i}^{\exp }}{\sigma_{i}}\right)^{2}+\sum_{i=1}^{4} \chi_{\mathrm{PMNS}, i}^{2}
$$

where $\sigma_{i}$ are the standard deviations of the experimental observables. The first term in eq. (2.17) includes the masses of quarks (6 observables) and charged leptons ( 3 observables) as well as the CKM parameters (4 observables) and the mass squared differences of the light neutrinos (2 observables). For these we use a Gaussian error.

The NuFit 3.0 results for the mass squared differences of the neutrinos are

$$
\begin{aligned}
& \Delta m_{21}^{2}=(7.50 \pm 0.18) \times 10^{-5} \mathrm{eV}^{2}, \\
& \Delta m_{31}^{2}=(2.524 \pm 0.0395) \times 10^{-3} \mathrm{eV}^{2} .
\end{aligned}
$$

The other Gaussian observables are listed in table 4 in the column ' $1 \mathrm{TeV}$ '. For reference we also show the corresponding results in the $\overline{\mathrm{MS}}$ scheme in table 3 .

The Gaussian treatment of the errors would be yet inadequate for the PMNS parameters. Therefore, for these we use the corresponding $\chi^{2}$-profiles from NuFit 3.0 [70], which are represented in the second term in eq. (2.17). Our implementation also includes a check 
for inverted or normal mass ordering to ensure the right distribution is used. In particular we also include the second (higher) $\chi^{2}$ minimum of $\theta_{23}^{l}$. In that way, also the CP phase $\delta_{\mathrm{CP}}^{l}$ can be included although it is not yet measured directly. Note that neutrino observables are treated here as tree-level observables and consequently by definition do not take part in the running under the renormalisation group. Considering this, we employ the results of NuFit 3.0 directly at $M_{\text {SUSY }}=1 \mathrm{TeV}$.

Altogether, we have 19 observables and 22 parameters in the fit, including the three threshold parameters $\epsilon_{b}, \epsilon_{q}$ and $\epsilon_{l}$. As these stem from 1-loop corrections, we allow for them the following ranges:

$$
\begin{aligned}
& -0.05 \leq \epsilon_{q} \leq 0.05, \\
& -0.10 \leq \epsilon_{b} \leq 0.10, \\
& -0.03 \leq \epsilon_{l} \leq 0.03,
\end{aligned}
$$

see, for example, refs. [54, 63, 69, 71]. The corrections to quarks are generally larger since they receive SUSY QCD corrections. Furthermore, $\epsilon_{b}$ receives another correction from the potentially large stop trilinear SUSY breaking coupling. One might worry here that the corrections become non-perturbative, since our scan allows, e.g., $\left|\epsilon_{b} \tan \beta\right|>1$. But this is not the case since there are no higher order corrections $\mathcal{O}\left(\left(\epsilon_{b} \tan \beta\right)^{n}\right)$ with $n \geq 2$, see, e.g., [72].

In order to perform the fits we link our C++ code to the Sbplx/Subplex [73, 74] and ISRES algorithms [73, 75, 76] implemented in the NLopt 2.4.2 library [73]. The extensive numerical fits are performed on the TTP computing cluster. As these are highly time consuming we do not include two minor online-updates of the NuFit results which appeared in the meantime.

\section{Results of the SUSY SO(10) fit to flavour data}

In the following, we present our results for the $\chi^{2}$ fit of SUSY SO(10) models to the quark and lepton flavour data given at $1 \mathrm{TeV}$ as described in section 2.5. First, we present the global minima, which show that threshold corrections are essential to get a good fit in our setup. Afterwards, we present the $\chi^{2}$-profiles for the SUSY threshold parameters.

\subsection{Comparison of the global minima}

We search for the global minima for three different values of $\tan \beta=10,38$ and 50 as well as both with and without SUSY threshold corrections so that we have six global minima in total. The results of our global minimisation can be found in table 5 . The table shows the minimal $\chi^{2}$ from our scan, the pulls of the SM observables as well as the predictions for the light neutrino masses and the needed value of the threshold corrections in case they are included. We define the pull for an observable $\mathcal{O}_{i}$ with a Gaussian error by

$$
\operatorname{pull}_{i}=\frac{\mathcal{O}_{i}^{\text {theo }}-\mathcal{O}_{i}^{\exp }}{\sigma_{i}} .
$$




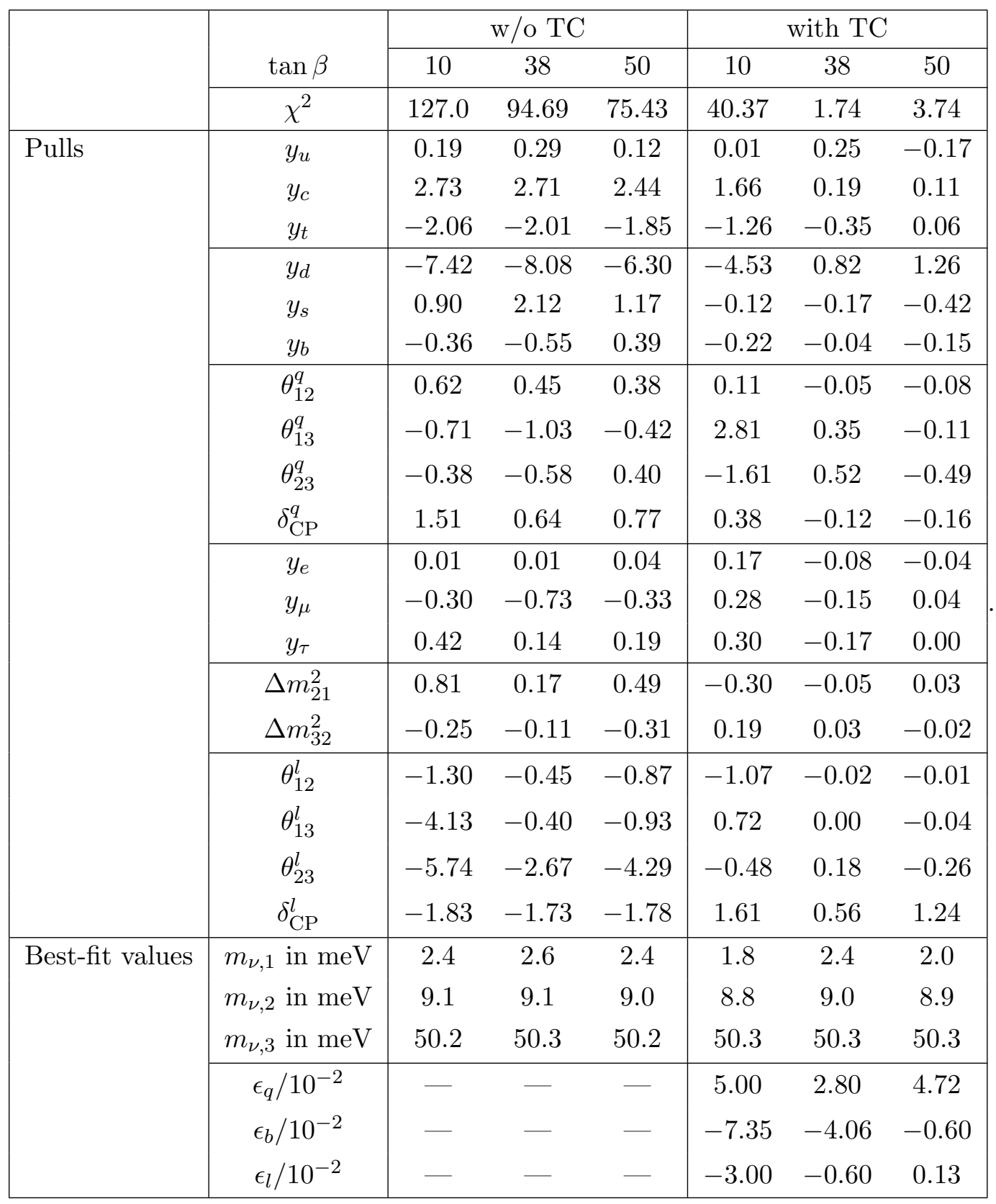

Table 5. Results of the global minimisation. Minimal $\chi^{2}$, pulls of the observables in the $\overline{\mathrm{DR}}$ scheme (for the definition of pulls see eqs. (3.1) and (3.2)) for different values of $\tan \beta$ as well as our best-fit values for the light neutrino masses and SUSY threshold corrections (TC) if included in the fit. 

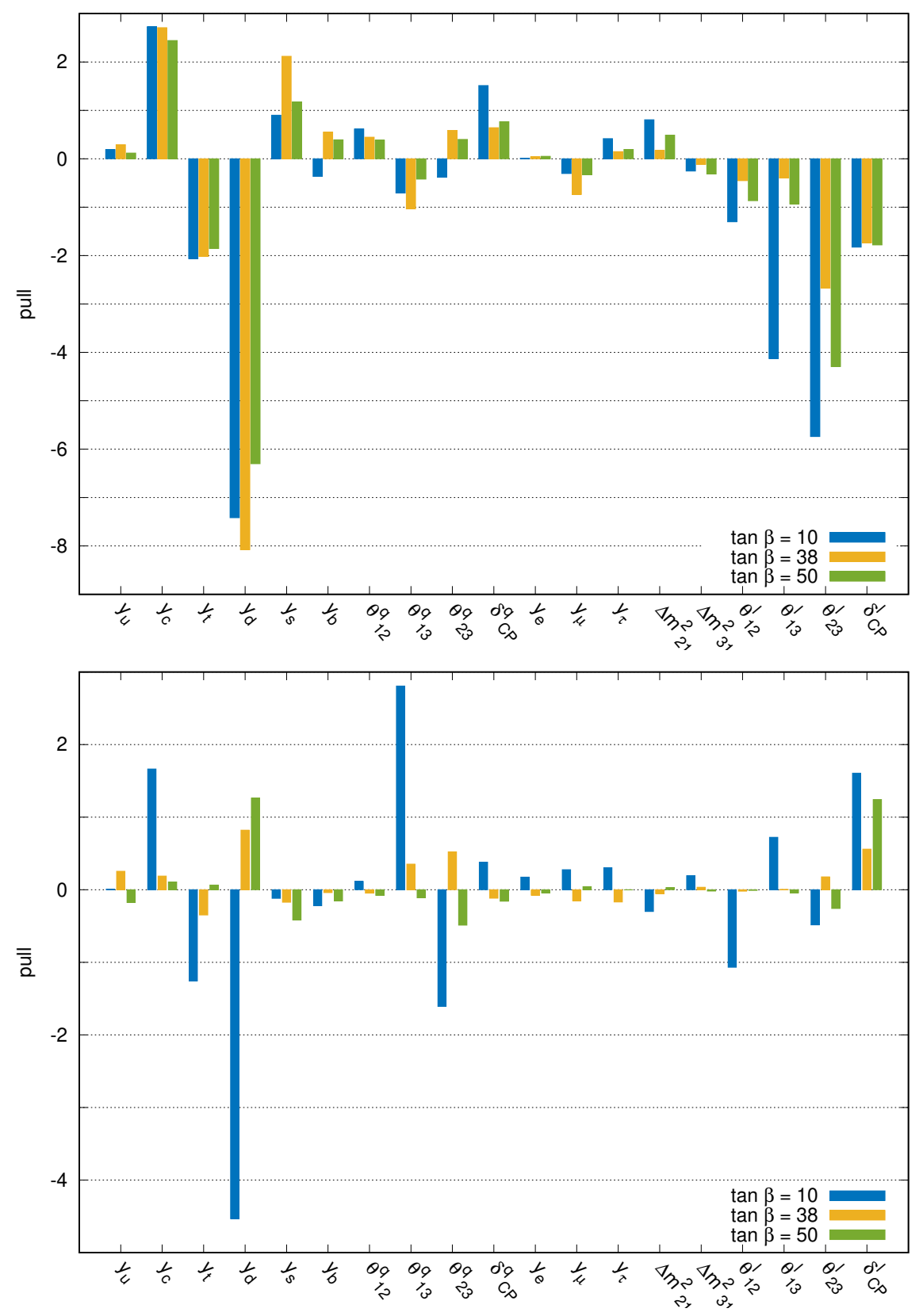

Figure 1. Results of the global minimisation. Pulls of the observables for different values of $\tan \beta$ without (top) and with (bottom) SUSY threshold corrections.

For the PMNS parameters we use the $\chi^{2}$-distribution and the best-fit values of NuFit $\mathcal{O}_{i}^{\text {best-fit }}$ to define

$$
\operatorname{pull}_{i}=\operatorname{sign}\left(\mathcal{O}_{i}^{\text {theo }}-\mathcal{O}_{i}^{\text {best}- \text { fit }}\right) \sqrt{\chi_{\text {PMNS }, \mathrm{i}}^{2}}
$$

Additionally, these pulls are also visualised in the bar charts of figure 1. The corresponding detailed fit parameters at the GUT scale are given in appendix B. 
Without including SUSY threshold corrections we find a poor description of the data by our $\mathrm{SO}(10)$ setup, with best fit points having $\chi^{2}>70$. It turns out that this is especially triggered by the high precision of quark mass determinations on the lattice, in particular, for the down-type quark Yukawa coupling the tension is for all three values of $\tan \beta$ more than $6 \sigma$. There is another major tension in the atmospheric mixing angle where the modulus of the pull is always larger than 2 and even larger than 5 for $\tan \beta=10$.

Note that the fit without threshold corrections has as many parameters as observables, and including threshold corrections we have three parameters more than observables so that one might expect to see a perfect fit. However, the dependence of the observables on the parameters is highly non-linear, so that the expectation of a perfect fit in this case is misleading. Indeed, the observables are singular values of complex matrices with a strong hierarchical structure and are coupled via non-linear differential equations. This also leads to the fact that not every GUT scale fit parameter enters the same low-scale observables. For instance, the 1-1 elements of the Yukawa matrices will practically not affect the masses of the third generation fermions, whereas the 3-3 elements affect all other couplings and together with the 2-3 elements determine the mixing between second and third generation.

Having more parameters than observables the statistical interpretation of $\chi^{2}$ is not straight forward. Nevertheless, we can still use the $\chi^{2}$ information in order to compare different fit scenarios. First of all, our results clearly prefer a large $\tan \beta$ which is a wellknown result. Our minimal $\mathrm{SO}(10)$ setup is hardly viable without threshold corrections and for $\tan \beta=10$ even including that corrections, see table 5. Therefore, we conclude that sizeable, i.e., percent order SUSY threshold corrections and large values of $\tan \beta$ are needed in order to be in accordance with data. Note that for $\tan \beta=10$ at the global minimum we have $\epsilon_{q}=0.05$ and $\epsilon_{l}=-0.03$ which are both at the edges of the respective allowed ranges for these parameters. That means the fit would prefer larger values of $\epsilon_{q}$ and $\epsilon_{l}$ or larger $\tan \beta$.

Comparing the pulls of the different observables, it is remarkable that besides $y_{d}$ we find large discrepancies in the neutrino observables. This makes a thorough treatment of the right-handed neutrinos necessary: we treat them in an effective field theory picture where each of them is integrated out at its mass scale, as described in detail in ref. [57].

Within our setup we always find the neutrinos to have a normal mass hierarchy with the heaviest neutrino having a mass of about $50 \mathrm{meV}$. The sum of light neutrino masses is below the cosmological bound $\sum m_{\nu} \lesssim 0.23 \mathrm{eV} / c^{2}$ [77] for all global minima. The same holds obviously for the effective $\beta$-decay mass

$$
m_{\nu, \beta}^{2}=\sum_{i=1}^{3}\left|V_{e i}^{l}\right|^{2} m_{\nu, i}^{2}
$$

where the current upper bound is $m_{\nu, \beta}<2.05 \mathrm{eV}$ [78]. Our best-fit results are also far below the sensitivity of KATRIN [79] which is expected to improve this bound by an order of magnitude. 

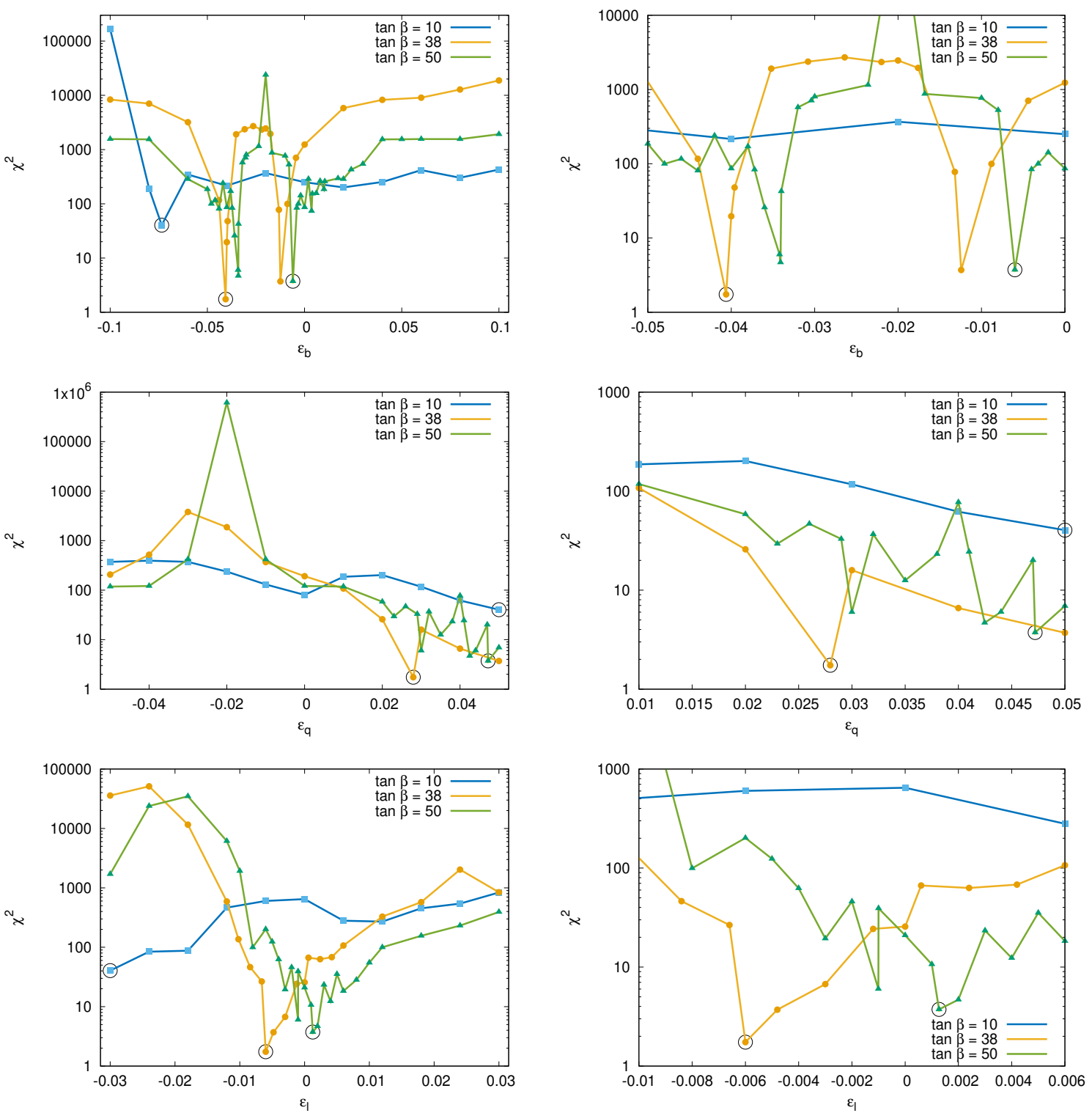

Figure 2. Results of the minimisation for fixed values of the threshold corrections. The circles mark the global minima. The left plots show the full range of the fit whereas the right plots are zoomed around the global minima.

\subsection{Likelihood profiles for threshold corrections}

Given the importance of the SUSY threshold corrections for the goodness of fit of SO(10) models to fermion masses, see section 3.1, it is an interesting question how sensitive the data is to the magnitude of the threshold corrections. This has in particular non-trivial implications for potential concrete SUSY spectra. In order to answer this question, we determine the $\chi^{2}$-profiles of $\epsilon_{b}, \epsilon_{q}$ and $\epsilon_{l}$, which are shown in figure 2. On the left-hand side we show the results over the full range that we allow, see eqs. (2.20)-(2.22), while on the right-hand side we zoom into the interesting regions around the minima for $\tan \beta=38,50$. Around 
the minima we increased the number of points to exclude possible numerical artefacts and to obtain a better resolution of the profile.

Keeping in mind that the threshold corrections get multiplied by $\tan \beta$, see eqs. (2.15) and (2.16), we first like to discuss the case of $\tan \beta=10$. In this case, the $\chi^{2}$-profiles have in general less features than for $\tan \beta=38,50$. Still, there is a distinct minimum in the $\epsilon_{b}$-profile and a clear preference for smaller values of $\epsilon_{l}$ and larger values of $\epsilon_{q}$. Actually, the global minimum lies on the border of the allowed ranges for $\epsilon_{q}$ and $\epsilon_{l}$ as we mentioned before.

Interestingly, for $\tan \beta=38$ and $\tan \beta=50$ we see two competing narrow minima in the $\chi^{2}$-profile of $\epsilon_{b}$. In fact, our fit fixes the viable values for $\epsilon_{b}$ to narrow intervals which can be an interesting input for SUSY model building and phenomenology. Furthermore, we obtain that $\epsilon_{b}<0$ whereas $\epsilon_{q}>0$. This means that the fit favours to decrease the bottom Yukawa coupling and to increase the Yukawa coupling for the strange quark and down quark.

As a curiosity, we note that in our scans the deeper of the two minima for $\tan \beta=38$ is the one with smaller $\epsilon_{b}$ while for $\tan \beta=50$ it is the other way around. In particular for $\tan \beta=50$ the minima are almost degenerate with $\chi^{2}=3.7$ and $\chi^{2}=4.7$. It might be an interesting observation for SUSY phenomenology, that the two minima competing correspond to either $\epsilon_{b} \tan \beta<-1$ or $-1<\epsilon_{b} \tan \beta<0$, respectively.

Altogether, it is interesting to note that the largest tensions, cf. figures 1 and 3 , are in the down quark mass, up quark mass, $\theta_{13}^{q}$ and the leptonic CP phase $\delta_{\mathrm{CP}}^{l}$. Especially, for the light quark masses and the leptonic CP phase we expect a significant reduction in the uncertainties in the next decade which might help to disfavour one of the two minima or even both minima. The importance of the leptonic CP phase for $\mathrm{SO}(10)$ fits was also noticed already, e.g., in ref. [52]. This again shows that, although we have more parameters than observables, we can still make quantitative statements.

\section{Summary and conclusions}

SUSY SO(10) is one of the best motivated extensions of the SM and due to its aesthetics it served as a guiding principle in particle physics for quite a while. Yet, as no signs of SUSY or proton decay have been seen in experimental searches, it still remains uncertain if SUSY GUTs are just a dream or if they have indeed anything to do with nature.

Fermion masses and mixing can give a partial answer to this question. In this work we have fitted a minimal $\mathrm{SO}(10)$ Yukawa sector to recent data for fermion masses and mixing. Regarding the available data, compared to most previous studies there is a quite significant reduction of uncertainties for light quark masses and neutrino mixing parameters. These changes are so drastic that minimal SUSY SO(10) without the inclusion of sizeable SUSY threshold corrections is highly disfavoured nowadays.

Although it was known for quite a while already that these corrections are generically large and important [4-7] they were usually neglected in fits. Even in our rather minimal setup the Yukawa sector has already 19 parameters which we fit to 19 observables. SUSY 

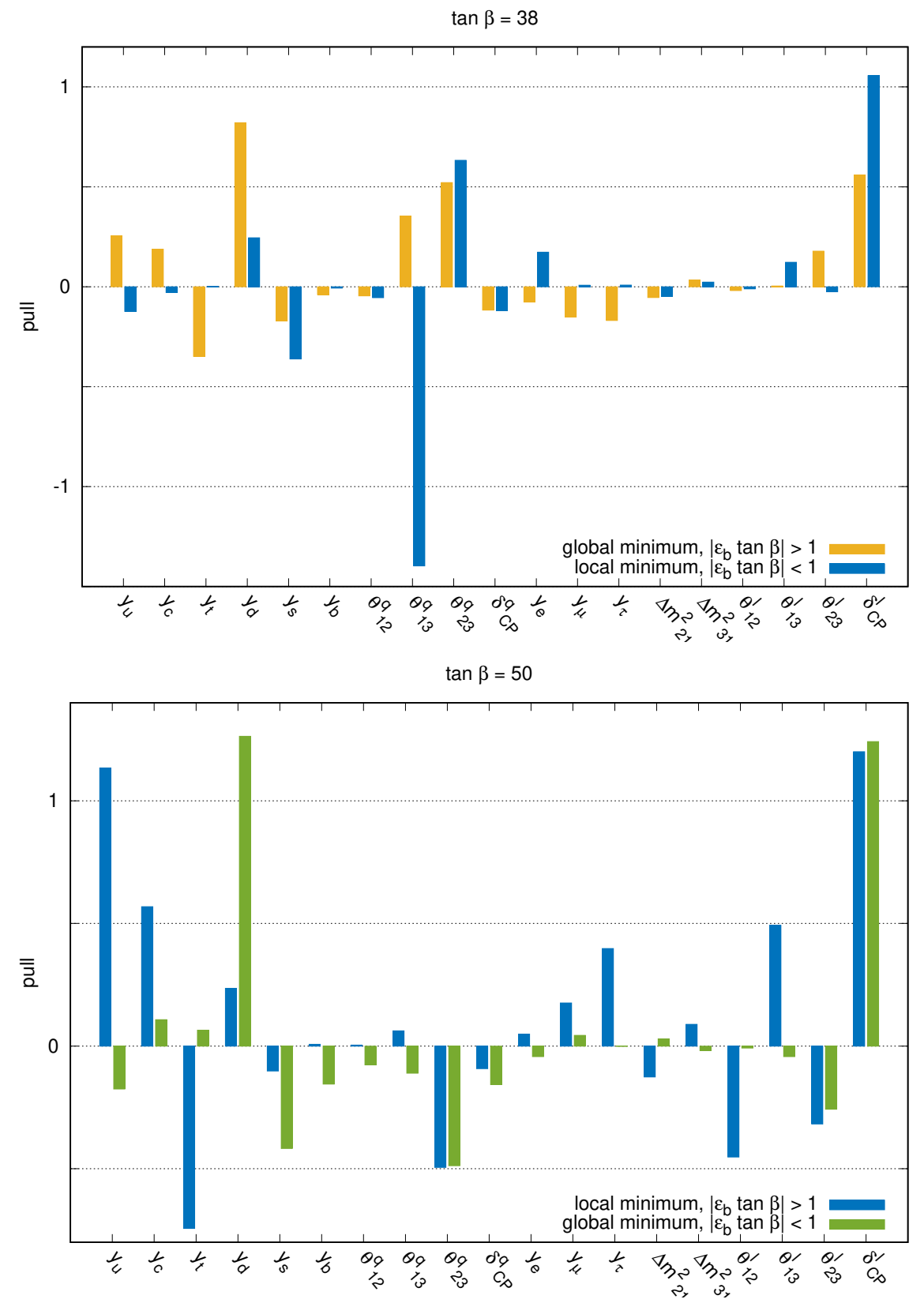

Figure 3. Comparison of global minima and local minima. Shown are the pulls of the observables corresponding to the narrow minima in the $\chi^{2}$ profiles of figure 2 . 
thresholds plausibly add at least another three parameters which we have included here increasing the computational needs quite drastically.

In fact, we did not only include them but also provide for them $\chi^{2}$-profiles for the first time to our knowledge. These turn out to be particularly interesting for the third generation parameter $\epsilon_{b}$. We find two rather narrow minima for $\tan \beta=38$ and 50 each, all of them with an acceptable $\chi^{2}<5$. These precise predictions for $\epsilon_{b}$ have consequences for the SUSY spectrum and phenomenology which nevertheless goes beyond the scope of the current work. Unfortunately, we could not identify here a general set of observables which allows us to distinguish between the two solutions for both $\tan \beta$ values. In principle, once the SUSY spectrum is known we can directly calculate the $\epsilon$-parameters which would provide a definite answer.

Until then a more precise determination of the light quark masses and neutrino mixing observables will help to challenge some of the minima which we have found here. The good thing is that we expect a significant progress for these observables in the next years before a detailed knowledge of the SUSY spectrum could realistically be available.

In particular, we would like to highlight here the role of the leptonic Dirac CP violating

phase $\delta_{\mathrm{CP}}^{l}$ which creates a considerable contribution to the $\chi^{2}$ for most of the found minima. Hence, with the measurement of low energy leptonic CP violation we can test SUSY SO(10).

In summary, we see that with the emergence of precision neutrino data and improved lattice calculations SUSY SO(10) can be challenged. So that in the near future we might find out, if we have been foolishly dreaming or following a path towards a deeper understanding of nature.

\section{Acknowledgments}

TD acknowledges support by the doctoral school GRK 1694 (DFG). SS is supported by a DFG Forschungsstipendium under contract no. SCHA 2125/1-1. MS is supported by the Ministry of Science and Technology (MOST) of Taiwan under grant number MOST 107-2112-M-007-031-MY3.

\section{A SM observables at various scales}

The quark masses (except for the top quark) and the value of $\alpha_{s}^{(5)}$ are taken from ref. [60], as described in section 2.3. For the mass of the top quark we use the PDG average $m_{t, \text { pole }}=174.2 \pm 1.4 \mathrm{GeV}$ [61]. At two-loop accuracy also the quartic Higgs coupling contributes to the RGE running of the Yukawas in the SM

$$
\lambda=\frac{2 m_{\text {Higgs }}}{v^{2}} .
$$

This convention is in accordance with the convention used in the two-loop RGEs, i.e. the quartic term in the SM Higgs potential is given by $\lambda\left(\phi^{\dagger} \phi\right)^{2} / 4$. We calculate $\lambda$ from the Higgs mass measurement $m_{\text {Higgs }}=125.09 \pm 0.24 \mathrm{GeV}[61$. 
The values for the charged lepton Yukawa couplings and the electroweak gauge couplings have already been given in ref. [63]

$$
\begin{aligned}
10^{6} \times Y_{e}\left(M_{Z}\right) & =2.794745 \pm 0.0000155 \\
10^{4} \times Y_{\mu}\left(M_{Z}\right) & =5.899863 \pm 0.0000185 \\
10^{2} \times Y_{\tau}\left(M_{Z}\right) & =1.002950 \pm 0.0000905 \\
g_{1}\left(M_{Z}\right) & =0.461425 \pm 0.0000435 \\
g_{2}\left(M_{Z}\right) & =0.65184 \pm 0.000175
\end{aligned}
$$

For the CKM matrix we use the updated results of the CKMfitter group as presented at ICHEP 2016 [64]. We translate from the Wolfenstein parametrisation to the parametrisation of the PDG and obtain

$$
\begin{aligned}
\theta_{12}^{q} & =0.227035 \pm 0.000293 \\
\theta_{13}^{q} & =0.003712 \pm 0.000139 \\
\theta_{23}^{q} & =0.0418112 \pm 0.000567 \\
\delta_{\mathrm{CP}}^{q} & =1.1430 \pm 0.0108
\end{aligned}
$$

In table 3 all these values are given at $1 \mathrm{TeV}, 3 \mathrm{TeV}$ and $10 \mathrm{TeV}$, respectively. The procedure of RGE running is described in section 2.3. For use within SUSY models the values have also been converted to the $\overline{\mathrm{DR}}$ scheme, cf. table 4 .

\section{B GUT scale parameters for the global minima}

For reference, we give here the coordinates of our global minima to a high numerical precision, which allows the reproduction of the results given in table 5. We determine the GUT scale by minimising the sum of the squared differences of the gauge couplings. The central values of the Yukawa couplings are included in the running to the GUT scale. At $M_{\mathrm{GUT}}=1.35276 \times 10^{16}$ we find

$$
g_{1}=0.706132, \quad g_{2}=0.708008, \quad g_{3}=0.705465
$$

In all fits, these values remain fixed. The parametrisation of the MSSM Yukawa matrices is given in eqs. (2.2)-(2.7). If SUSY threshold corrections are included, their value at $M_{\mathrm{SUSY}}$ is also given below. The parametrisation of the $\mathrm{SO}(10)$ Yukawa couplings is

$$
Y_{10}=\operatorname{diag}\left(H_{1}, H_{2}, H_{3}\right), \quad Y_{126}=\left(\begin{array}{lll}
F_{1} & F_{2} & F_{3} \\
F_{2} & F_{4} & F_{5} \\
F_{3} & F_{5} & F_{6}
\end{array}\right) .
$$

\section{B.1 Global minima without SUSY threshold corrections}

We list here our fit results for the coordinates of the global minima without SUSY threshold corrections for different values of $\tan \beta$. For reference, we use a high numerical precision. 
$\tan \beta=10$, no threshold corrections, $\chi^{2}=127.016$

$$
\begin{aligned}
r & =8.93109, & s & =0.40219+0.0537018 \mathrm{i}, \\
r_{R} & =5.7222 \times 10^{14} \mathrm{GeV}, & H_{1} & =-8.13678 \times 10^{-6}, \\
H_{2} & =0.000546749, & H_{3} & =0.0587967, \\
F_{1} & =(3.62943+1.11577 \mathrm{i}) \times 10^{-5}, & F_{2} & =(-4.86448-6.8181 \mathrm{i}) \times 10^{-5}, \\
F_{3} & =-8.669 \times 10^{-5}+0.00101114 \mathrm{i}, & F_{4} & =-0.00122229+0.000483037 \mathrm{i}, \\
F_{5} & =0.00229999-0.00243838 \mathrm{i}, & F_{6} & =-0.00436435+0.000144527 \mathrm{i} .
\end{aligned}
$$

$\tan \beta=38$, no threshold corrections, $\chi^{2}=94.6859$

$$
\begin{aligned}
r & =2.09333, & s & =0.361579+0.00221939 \mathrm{i}, \\
r_{R} & =-1.01454 \times 10^{14} \mathrm{GeV}, & H_{1} & =-3.76306 \times 10^{-5}, \\
H_{2} & =0.0017972, & H_{3} & =0.254405, \\
F_{1} & =0.000100472+0.000137311 \mathrm{i}, & F_{2} & =3.1103 \times 10^{-5}+0.000457557 \mathrm{i}, \\
F_{3} & =-0.00260288+0.0039351 \mathrm{i}, & F_{4} & =-0.00474944+0.00161864 \mathrm{i}, \\
F_{5} & =-0.0107501+0.00940057 \mathrm{i}, & & F_{6}=-0.0119578-0.047225 \mathrm{i} .
\end{aligned}
$$

$\tan \beta=50$, no threshold corrections, $\chi^{2}=75.428$

$$
\begin{aligned}
r & =-1.351, & s & =0.380723+0.0112833 \mathrm{i}, \\
r_{R} & =7.08356 \times 10^{13} \mathrm{GeV}, & H_{1} & =-5.44756 \times 10^{-5}, \\
H_{2} & =0.00295336, & H_{3} & =0.418203, \\
F_{1} & =0.000198815+0.000167965 \mathrm{i}, & F_{2} & =0.000197095+0.00060758 \mathrm{i}, \\
F_{3} & =0.00302059-0.00664685 \mathrm{i}, & F_{4} & =-0.00713153+0.00251093 \mathrm{i}, \\
F_{5} & =0.0167445-0.0153386 \mathrm{i}, & F_{6} & =-0.0253209-0.0530162 \mathrm{i} .
\end{aligned}
$$

\section{B.2 Global minima with SUSY threshold corrections}

Here we give our fit results for the coordinates of the global minima including SUSY threshold corrections, again with a high numerical precision, see above.

$\tan \beta=10$, with threshold corrections, $\chi^{2}=40.3671$

$$
\begin{aligned}
r & =2.68759, \\
r_{R} & =4.05262 \times 10^{13} \mathrm{GeV}, \\
H_{2} & =0.000694187, \\
F_{1} & =(-7.52986-11.2321 \mathrm{i}) \times 10^{-6}, \\
F_{3} & =0.000578945+0.000363829 \mathrm{i}, \\
F_{5} & =0.0100828+0.000256401 \mathrm{i}, \\
\epsilon_{q} & =0.05000 \\
\epsilon_{l} & =-0.02999953 .
\end{aligned}
$$$$
s=0.146006-0.258778 \mathrm{i},
$$$$
H_{1}=5.28137 \times 10^{-6} \text {, }
$$$$
H_{3}=0.186542 \text {, }
$$$$
F_{2}=(-5.92321-9.76299 \mathrm{i}) \times 10^{-5},
$$$$
F_{4}=6.89316 \times 10^{-5}-0.000631902 \mathrm{i} \text {, }
$$$$
F_{6}=0.0424372+0.028391 \mathrm{i} \text {, }
$$$$
\epsilon_{b}=-0.07348963 \text {, }
$$ 
$\tan \beta=38$, with threshold corrections, $\chi^{2}=1.74389$

$$
\begin{aligned}
r & =0.739487, & s & =0.171775-0.0527206 \mathrm{i}, \\
r_{R} & =3.00163 \times 10^{12} \mathrm{GeV}, & H_{1} & =1.74192 \times 10^{-5}, \\
H_{2} & =0.00263311, & H_{3} & =0.873783, \\
F_{1} & =(-4.52508-1.40042) \times 10^{-5} \mathrm{i}, & F_{2} & =-0.000670712-0.000352788 \mathrm{i}, \\
F_{3} & =0.00257905+0.000499293 \mathrm{i}, & F_{4} & =-0.00325008-0.0013532 \mathrm{i}, \\
F_{5} & =0.0128581+0.0601288 \mathrm{i}, & F_{6} & =0.474974+0.216717 \mathrm{i}, \\
\epsilon_{q} & =0.02796541, & \epsilon_{b} & =-0.04061278, \\
\epsilon_{l} & =-0.006000 . & &
\end{aligned}
$$

$\tan \beta=50$, with threshold corrections, $\chi^{2}=3.73552$

$$
\begin{aligned}
r & =0.699764, & s & =0.137602-0.0279775 \mathrm{i}, \\
r_{R} & =2.33 \times 10^{12} \mathrm{GeV}, & H_{1} & =1.21069 \times 10^{-5}, \\
H_{2} & =0.00246264, & H_{3} & =0.988718, \\
F_{1} & =(-4.09545-1.24086 \mathrm{i}) \times 10^{-5}, & F_{2} & =0.000472532+0.00031884 \mathrm{i}, \\
F_{3} & =0.00483506+0.00119957 \mathrm{i}, & F_{4} & =-0.00190458+0.00028723 \mathrm{i}, \\
F_{5} & =-0.0316564-0.0584933 \mathrm{i}, & F_{6} & =0.607543+0.0633526 \mathrm{i}, \\
\epsilon_{q} & =0.04720754, & \epsilon_{b} & =-0.006000, \\
\epsilon_{l} & =0.001271233 . & &
\end{aligned}
$$

\section{B.3 Local minima with SUSY threshold corrections}

Here we give our fit results for the coordinates of the local minima of $\epsilon_{b}$ including SUSY threshold corrections, again with a high numerical precision, see above.

$\tan \beta=38$, with threshold corrections, $\chi^{2}=3.71108$

$$
\begin{aligned}
r & =0.670043 \\
r_{R} & =2.03128 \times 10^{12} \mathrm{GeV} \\
H_{2} & =0.00255376 \\
F_{1} & =-3.38058 \times 10^{-5}-5.62988 \times 10^{-10} \mathrm{i}, \\
F_{3} & =0.00523839+0.000883983 \mathrm{i} \\
F_{5} & =0.0259931+0.063712 \mathrm{i} \\
\epsilon_{q} & =0.04999992 \\
\epsilon_{l} & =-0.0048000
\end{aligned}
$$

$$
\begin{aligned}
s & =0.11989-0.0413042 \mathrm{i}, \\
H_{1} & =1.09545 \times 10^{-5}, \\
H_{3} & =1.05489 \\
F_{2} & =-0.00040558-0.000269377 \mathrm{i}, \\
F_{4} & =-0.00226047+0.000662944 \mathrm{i}, \\
F_{6} & =0.622678+0.0958844 \mathrm{i}, \\
\epsilon_{b} & =-0.01243252
\end{aligned}
$$


$\tan \beta=50$, with threshold corrections, $\chi^{2}=4.6864$

$$
\begin{aligned}
r & =0.719454, & s & =0.17033-0.0410235 \mathrm{i}, \\
r_{R} & =2.89057 \times 10^{12} \mathrm{GeV}, & H_{1} & =1.14972 \times 10^{-5}, \\
H_{2} & =0.00276048, & H_{3} & =0.898288, \\
F_{1} & =(-3.87624-1.31146 \mathrm{i}) \times 10^{-5}, & F_{2} & =-0.000600001-0.000317934 \mathrm{i} \\
F_{3} & =-0.00275464-0.00102727 \mathrm{i}, & F_{4} & =-0.00375989-0.00115394 \mathrm{i} \\
F_{5} & =-0.0164547-0.0618966 \mathrm{i}, & F_{6} & =0.552+0.0273906 \mathrm{i} \\
\epsilon_{q} & =0.4246740, & \epsilon_{b} & =-0.3408847, \\
\epsilon_{l} & =0.0020000 . & &
\end{aligned}
$$

Open Access. This article is distributed under the terms of the Creative Commons Attribution License (CC-BY 4.0), which permits any use, distribution and reproduction in any medium, provided the original author(s) and source are credited.

\section{References}

[1] H. Georgi, The State of the Art - Gauge Theories, AIP Conf. Proc. 23 (1975) 575.

[2] H. Fritzsch and P. Minkowski, Unified Interactions of Leptons and Hadrons, Annals Phys. 93 (1975) 193 [INSPIRE].

[3] U. Amaldi, W. de Boer and H. Furstenau, Comparison of grand unified theories with electroweak and strong coupling constants measured at LEP, Phys. Lett. B 260 (1991) 447 [INSPIRE].

[4] L.J. Hall, R. Rattazzi and U. Sarid, The top quark mass in supersymmetric $\mathrm{SO}(10)$ unification, Phys. Rev. D 50 (1994) 7048 [hep-ph/9306309] [INSPIRE].

[5] M. Carena, M. Olechowski, S. Pokorski and C.E.M. Wagner, Electroweak symmetry breaking and bottom-top Yukawa unification, Nucl. Phys. B 426 (1994) 269 [hep-ph/9402253] [INSPIRE].

[6] R. Hempfling, Yukawa coupling unification with supersymmetric threshold corrections, Phys. Rev. D 49 (1994) 6168 [INSPIRE].

[7] T. Blazek, S. Raby and S. Pokorski, Finite supersymmetric threshold corrections to CKM matrix elements in the large $\tan \beta$ regime, Phys. Rev. D 52 (1995) 4151 [hep-ph/9504364] [INSPIRE].

[8] C.S. Aulakh and R.N. Mohapatra, Implications of Supersymmetric $\mathrm{SO}(10)$ Grand Unification, Phys. Rev. D 28 (1983) 217 [InSPIRE].

[9] T.E. Clark, T.-K. Kuo and N. Nakagawa, A SO(10) Supersymmetric Grand Unified Theory, Phys. Lett. B 115 (1982) 26 [InSPIRE].

[10] B. Ananthanarayan, G. Lazarides and Q. Shafi, Top mass prediction from supersymmetric guts, Phys. Rev. D 44 (1991) 1613 [InSPIRE].

[11] K.S. Babu and R.N. Mohapatra, Predictive neutrino spectrum in minimal $\mathrm{SO}(10)$ grand unification, Phys. Rev. Lett. 70 (1993) 2845 [hep-ph/9209215] [INSPIRE]. 
[12] L. Lavoura, Predicting the neutrino spectrum in minimal $\mathrm{SO}(10)$ grand unification, Phys. Rev. D 48 (1993) 5440 [hep-ph/9306297] [INSPIRE].

[13] F. Vissani and A. Yu. Smirnov, Neutrino masses and b- $\tau$ unification in the supersymmetric standard model, Phys. Lett. B 341 (1994) 173 [hep-ph/9405399] [INSPIRE].

[14] B. Brahmachari and R.N. Mohapatra, Unified explanation of the solar and atmospheric neutrino puzzles in a minimal supersymmetric $\mathrm{SO}(10)$ model, Phys. Rev. D 58 (1998) 015001 [hep-ph/9710371] [INSPIRE].

[15] K. Matsuda, T. Fukuyama and H. Nishiura, $\mathrm{SO}(10)$ GUT and quark lepton mass matrices, Phys. Rev. D 61 (2000) 053001 [hep-ph/9906433] [InSPIRE].

[16] C.S. Aulakh, B. Bajc, A. Melfo, A. Rasin and G. Senjanović, SO(10) theory of R-parity and neutrino mass, Nucl. Phys. B 597 (2001) 89 [hep-ph/0004031] [InSPIRE].

[17] B. Bajc, G. Senjanović and F. Vissani, How neutrino and charged fermion masses are connected within minimal supersymmetric SO(10), PoS (HEP2001) 198 (2001) [hep-ph/0110310] [INSPIRE].

[18] T. Fukuyama and N. Okada, Neutrino oscillation data versus minimal supersymmetric SO(10) model, JHEP 11 (2002) 011 [hep-ph/0205066] [INSPIRE].

[19] N. Oshimo, Antisymmetric Higgs representation in $\mathrm{SO}(10)$ for neutrinos, Phys. Rev. D 66 (2002) 095010 [hep-ph/0206239] [INSPIRE].

[20] B. Bajc, G. Senjanović and F. Vissani, $b$ - $\tau$ unification and large atmospheric mixing: A case for noncanonical seesaw, Phys. Rev. Lett. 90 (2003) 051802 [hep-ph/0210207] [INSPIRE].

[21] H.S. Goh, R.N. Mohapatra and S.-P. Ng, Minimal SUSY SO(10), b- $\tau$ unification and large neutrino mixings, Phys. Lett. B 570 (2003) 215 [hep-ph/0303055] [INSPIRE].

[22] N. Oshimo, Model for neutrino mixing based on SO(10), Nucl. Phys. B 668 (2003) 258 [hep-ph/0305166] [INSPIRE].

[23] C.S. Aulakh, B. Bajc, A. Melfo, G. Senjanović and F. Vissani, The minimal supersymmetric grand unified theory, Phys. Lett. B 588 (2004) 196 [hep-ph/0306242] [INSPIRE].

[24] H.S. Goh, R.N. Mohapatra and S.-P. Ng, Minimal SUSY SO(10) model and predictions for neutrino mixings and leptonic CP-violation, Phys. Rev. D 68 (2003) 115008 [hep-ph/0308197] [INSPIRE].

[25] B. Bajc, A. Melfo, G. Senjanović and F. Vissani, The minimal supersymmetric grand unified theory. 1. Symmetry breaking and the particle spectrum, Phys. Rev. D 70 (2004) 035007 [hep-ph/0402122] [INSPIRE].

[26] B. Bajc, G. Senjanović and F. Vissani, Probing the nature of the seesaw in renormalizable SO(10), Phys. Rev. D 70 (2004) 093002 [hep-ph/0402140] [INSPIRE].

[27] C.S. Aulakh and A. Girdhar, $\mathrm{SO}(10)$ MSGUT: Spectra, couplings and threshold effects, Nucl. Phys. B 711 (2005) 275 [hep-ph/0405074] [INSPIRE].

[28] S. Bertolini, M. Frigerio and M. Malinsky, Fermion masses in SUSY SO(10) with type-II seesaw: A non-minimal predictive scenario, Phys. Rev. D 70 (2004) 095002 [hep-ph/0406117] [INSPIRE].

[29] B. Dutta, Y. Mimura and R.N. Mohapatra, Neutrino masses and mixings in a predictive SO(10) model with CKM CP-violation, Phys. Lett. B 603 (2004) 35 [hep-ph/0406262] [INSPIRE]. 
[30] S. Bertolini and M. Malinsky, On CP-violation in minimal renormalizable SUSY $\mathrm{SO}(10)$ and beyond, Phys. Rev. D 72 (2005) 055021 [hep-ph/0504241] [INSPIRE].

[31] K.S. Babu and C. Macesanu, Neutrino masses and mixings in a minimal $\mathrm{SO}(10)$ model, Phys. Rev. D 72 (2005) 115003 [hep-ph/0505200] [INSPIRE].

[32] B. Bajc, A. Melfo, G. Senjanović and F. Vissani, Fermion mass relations in a supersymmetric SO(10) theory, Phys. Lett. B 634 (2006) 272 [hep-ph/0511352] [INSPIRE].

[33] C.S. Aulakh and S.K. Garg, MSGUT: From bloom to doom, Nucl. Phys. B 757 (2006) 47 [hep-ph/0512224] [INSPIRE].

[34] S. Bertolini, T. Schwetz and M. Malinsky, Fermion masses and mixings in $\mathrm{SO}(10)$ models and the neutrino challenge to SUSY GUTs, Phys. Rev. D 73 (2006) 115012 [hep-ph/0605006] [INSPIRE].

[35] W. Grimus and H. Kuhbock, Fermion masses and mixings in a renormalizable $\mathrm{SO}(10) \times Z_{2}$ GUT, Phys. Lett. B 643 (2006) 182 [hep-ph/0607197] [INSPIRE].

[36] C.S. Aulakh and S.K. Garg, The New Minimal Supersymmetric GUT: Spectra, RG analysis and fitting formulae, hep-ph/0612021 [INSPIRE].

[37] T. Fukuyama, K. Matsuda and H. Nishiura, Zero texture model and $\mathrm{SO}(10)$ GUT, Int. J. Mod. Phys. A 22 (2007) 5325 [hep-ph/0702284] [INSPIRE].

[38] G. Ross and M. Serna, Unification and fermion mass structure, Phys. Lett. B 664 (2008) 97 [arXiv: 0704.1248] [INSPIRE].

[39] C.S. Aulakh, Pinning down the new minimal supersymmetric GUT, Phys. Lett. B 661 (2008) 196 [arXiv:0710.3945] [InSPIRE].

[40] C.S. Aulakh and S.K. Garg, Correcting $\alpha_{3}\left(M_{Z}\right)$ in the NMSGUT, Mod. Phys. Lett. A 24 (2009) 1711 [arXiv:0710.4018] [INSPIRE].

[41] C.S. Aulakh and S.K. Garg, The New Minimal Supersymmetric GUT: Spectra, RG analysis and Fermion Fits, Nucl. Phys. B 857 (2012) 101 [arXiv:0807.0917] [inSPIRE].

[42] B. Bajc, I. Dorsner and M. Nemevšek, Minimal SO(10) splits supersymmetry, JHEP 11 (2008) 007 [arXiv:0809.1069] [InSPIRE].

[43] G. Altarelli and G. Blankenburg, Different $\mathrm{SO}(10)$ Paths to Fermion Masses and Mixings, JHEP 03 (2011) 133 [arXiv: 1012.2697] [INSPIRE].

[44] A.S. Joshipura and K.M. Patel, Fermion Masses in SO(10) Models, Phys. Rev. D 83 (2011) 095002 [arXiv: 1102 .5148] [INSPIRE].

[45] P.S. Bhupal Dev, R.N. Mohapatra and M. Severson, Neutrino Mixings in $\mathrm{SO}(10)$ with Type II Seesaw and $\theta_{13}$, Phys. Rev. D 84 (2011) 053005 [arXiv:1107.2378] [InSPIRE].

[46] P.S. Bhupal Dev, B. Dutta, R.N. Mohapatra and M. Severson, $\theta_{13}$ and Proton Decay in a Minimal $\mathrm{SO}(10) \times S_{4}$ model of Flavor, Phys. Rev. D 86 (2012) 035002 [arXiv:1202.4012] [INSPIRE].

[47] F. Buccella, D. Falcone, C.S. Fong, E. Nardi and G. Ricciardi, Squeezing out predictions with leptogenesis from $\mathrm{SO}(10)$, Phys. Rev. D 86 (2012) 035012 [arXiv: 1203.0829] [INSPIRE].

[48] S. Dev, S. Kumar, S. Verma, S. Gupta and R.R. Gautam, Four Zero Texture Fermion Mass Matrices in SO(10) GUT, Eur. Phys. J. C 72 (2012) 1940 [arXiv:1203.1403] [InSPIRE]. 
[49] G. Altarelli and D. Meloni, A non supersymmetric $\mathrm{SO}(10)$ grand unified model for all the physics below $M_{\mathrm{GUT}}$, JHEP 08 (2013) 021 [arXiv: 1305.1001] [INSPIRE].

[50] A. Dueck and W. Rodejohann, Fits to SO(10) Grand Unified Models, JHEP 09 (2013) 024 [arXiv: 1306.4468] [INSPIRE].

[51] K.S. Babu, B. Bajc and S. Saad, Yukawa Sector of Minimal SO(10) Unification, JHEP 02 (2017) 136 [arXiv:1612.04329] [INSPIRE].

[52] K.S. Babu, B. Bajc and S. Saad, Resurrecting Minimal Yukawa Sector of SUSY SO(10), JHEP 10 (2018) 135 [arXiv: 1805.10631] [INSPIRE].

[53] A. Freitas, E. Gasser and U. Haisch, Supersymmetric large $\tan \beta$ corrections to $\Delta M_{d, s}$ and $B_{d, s} \rightarrow \mu^{+} \mu^{-}$revisited, Phys. Rev. D 76 (2007) 014016 [hep-ph/0702267] [INSPIRE].

[54] S. Antusch and M. Spinrath, Quark and lepton masses at the GUT scale including SUSY threshold corrections, Phys. Rev. D 78 (2008) 075020 [arXiv:0804.0717] [INSPIRE].

[55] S.F. King, Unified Models of Neutrinos, Flavour and CP-violation, Prog. Part. Nucl. Phys. 94 (2017) 217 [arXiv: 1701.04413] [INSPIRE].

[56] S. Antusch and M. Ratz, Supergraph techniques and two loop $\beta$-functions for renormalizable and nonrenormalizable operators, JHEP 07 (2002) 059 [hep-ph/0203027] [INSPIRE].

[57] S. Antusch, J. Kersten, M. Lindner, M. Ratz and M.A. Schmidt, Running neutrino mass parameters in see-saw scenarios, JHEP 03 (2005) 024 [hep-ph/0501272] [INSPIRE].

[58] K. Ahnert and M. Mulansky, Odeint - Solving Ordinary Differential Equations in $C++$, AIP Conf. Proc. 1389 (2011) 001.

[59] G. Guennebaud et al., Eigen v3, http://eigen.tuxfamily.org, (2010).

[60] S. Aoki et al., Review of lattice results concerning low-energy particle physics, Eur. Phys. J. C 77 (2017) 112 [arXiv:1607.00299] [INSPIRE].

[61] Particle Data Group collaboration, C. Patrignani et al., Review of Particle Physics, Chin. Phys. C 40 (2016) 100001 [inSPIRE].

[62] K.G. Chetyrkin, J.H. Kuhn and M. Steinhauser, RunDec: A Mathematica package for running and decoupling of the strong coupling and quark masses, Comput. Phys. Commun. 133 (2000) 43 [hep-ph/0004189] [INSPIRE].

[63] S. Antusch and V. Maurer, Running quark and lepton parameters at various scales, JHEP 11 (2013) 115 [arXiv: 1306.6879] [INSPIRE].

[64] CKMfitter Group collaboration, J. Charles et al., CP violation and the CKM matrix: Assessing the impact of the asymmetric B factories, Eur. Phys. J. C 41 (2005) 1 [hep-ph/0406184] [INSPIRE].

[65] UTfit collaboration, M. Bona et al., The 2004 UTfit collaboration report on the status of the unitarity triangle in the standard model, JHEP 07 (2005) 028 [hep-ph/0501199] [INSPIRE].

[66] M.E. Machacek and M.T. Vaughn, Two Loop Renormalization Group Equations in a General Quantum Field Theory. 2. Yukawa Couplings, Nucl. Phys. B 236 (1984) 221 [InSPIRE].

[67] S.P. Martin and M.T. Vaughn, Regularization dependence of running couplings in softly broken supersymmetry, Phys. Lett. B 318 (1993) 331 [hep-ph/9308222] [INSPIRE].

[68] M. Spinrath, New Aspects of Flavour Model Building in Supersymmetric Grand Unification, arXiv: 1009.2511 [INSPIRE]. 
[69] S. Antusch, L. Calibbi, V. Maurer and M. Spinrath, From Flavour to SUSY Flavour Models, Nucl. Phys. B 852 (2011) 108 [arXiv:1104.3040] [InSPIRE].

[70] I. Esteban, M.C. Gonzalez-Garcia, M. Maltoni, I. Martinez-Soler and T. Schwetz, Updated fit to three neutrino mixing: exploring the accelerator-reactor complementarity, JHEP 01 (2017) 087 [arXiv: 1611.01514] [INSPIRE].

[71] S. Antusch and M. Spinrath, New GUT predictions for quark and lepton mass ratios confronted with phenomenology, Phys. Rev. D 79 (2009) 095004 [arXiv:0902.4644] [INSPIRE].

[72] M. Carena, D. Garcia, U. Nierste and C.E.M. Wagner, Effective Lagrangian for the $\bar{t} b H^{+}$ interaction in the MSSM and charged Higgs phenomenology, Nucl. Phys. B 577 (2000) 88 [hep-ph/9912516] [INSPIRE].

[73] S.G. Johnson, The NLopt nonlinear-optimization package, http://ab-initio.mit.edu/nlopt.

[74] T. Rowan, Functional Stability Analysis of Numerical Algorithms, Ph.D. Thesis, Department of Computer Sciences, University of Texas at Austin, U.S.A., (1990).

[75] T.P. Runarsson and X. Yao, Search biases in constrained evolutionary optimization, IEEE Trans. Syst. Man Cybern. 35 (2005) 233.

[76] T.P. Runarsson and X. Yao, Stochastic ranking for constrained evolutionary optimization, IEEE Trans. Evol. Comput. 4 (2000) 284.

[77] Planck collaboration, P.A.R. Ade et al., Planck 2015 results. XIII. Cosmological parameters, Astron. Astrophys. 594 (2016) A13 [arXiv:1502.01589] [INSPIRE].

[78] Troitsk collaboration, V.N. Aseev et al., An upper limit on electron antineutrino mass from Troitsk experiment, Phys. Rev. D 84 (2011) 112003 [arXiv:1108.5034] [INSPIRE].

[79] KATRIN collaboration, A. Osipowicz et al., KATRIN: A next generation tritium beta decay experiment with sub-eV sensitivity for the electron neutrino mass. Letter of intent, hep-ex/0109033 [INSPIRE]. 